\title{
Reflets
}

Revue d'intervention sociale et communautaire

\section{Réflexion sur les comités consultatifs jeunesse}

\section{Nadine Lamoureux}

Volume 19, numéro 1, printemps 2013

URI : https://id.erudit.org/iderudit/1018050ar

DOI : https://doi.org/10.7202/1018050ar

Aller au sommaire du numéro

Éditeur(s)

Reflets, Revue d'intervention sociale et communautaire

ISSN

1203-4576 (imprimé)

1712-8498 (numérique)

Découvrir la revue

Citer cet article

Lamoureux, N. (2013). Réflexion sur les comités consultatifs jeunesse. Reflets, 19(1), 243-251. https://doi.org/10.7202/1018050ar

\section{Résumé de l'article}

Les comités consultatifs prennent plus d'importance au sein des organismes sociaux et deviennent un type d'intervention privilégié dans leur travail, notamment auprès de la jeunesse. En évoquant son expérience personnelle, l'auteure de cet article ouvre certaines pistes de réflexion par rapport à l'utilisation de tels comités dans une optique d'approche structurelle, à leurs bienfaits et à certaines de leurs limites.
Tous droits réservés (C) Reflets, Revue d'intervention sociale et communautaire, 2013 document est protégé par la loi sur le droit d'auteur. L’utilisation des services d'Érudit (y compris la reproduction) est assujettie à sa politique d'utilisation que vous pouvez consulter en ligne.

https://apropos.erudit.org/fr/usagers/politique-dutilisation/ 


\section{Réflexion sur les comités consultatifs jeunesse}

\section{Nadine Lamoureux}

Étudiante à la maîtrise, École de service social, Université d'Ottawa

\section{Résumé}

Les comités consultatifs prennent plus d'importance au sein des organismes sociaux et deviennent un type d'intervention privilégié dans leur travail, notamment auprès de la jeunesse. En évoquant son expérience personnelle, l'auteure de cet article ouvre certaines pistes de réflexion par rapport à l'utilisation de tels comités dans une optique d'approche structurelle, à leurs bienfaits et à certaines de leurs limites.

\section{Introduction}

"Je me suis questionnée sur le rôle qu'a pu jouer dans ma vie un comité consultatif semblable auquel j'ai participé durant mon adolescence."
Les comités consultatifs peuvent être un outil important d'intervention structurelle, en ce qu'ils permettent aux gens qui ne seraient généralement pas consultés d'être entendus. L'année dernière, lors d'un premier stage de maitrise en service social,j'ai travaillé avec le comité consultatif jeunesse (CCJ) d'un organisme qui emploie des principes du pour et par en santé mentale pour les jeunes et qui tente de collaborer étroitement avec son CCJ. Cette expérience m'a amenée à réfléchir sur l'impact que de tels comités peuvent avoir sur leurs membres. Je me suis questionnée sur le rôle qu'a pu jouer dans ma vie un comité consultatif semblable auquel j'ai participé durant mon adolescence. Le présent article a pour fondement mon expérience donc tant personnelle que professionnelle. Je tenterai d'encadrer mon questionnement et d'offrir quelques pistes de réflexion par rapport aux bienfaits 
et aux défis de la consultation comme modèle d'intervention structurelle auprès des jeunes.

Récemment, les organismes qui travaillent auprès des populations marginalisées ont entrepris de consulter davantage la population par rapport à leurs services et à leurs programmes (Hohenemster et Marshall, 2002). Cela s'explique par le fait que les organismes subventionnaires imposent souvent comme condition de financement la consultation de la population bénéficiaire de services (Thurston, et collab., 2004). Or, non seulement cette tendance à la consultation des participants s'accorde-t-elle avec la mission des CCJ, mais elle en influence aussi leur essor. En effet, en plus d'aider les organismes ou agences à connaitre les besoins de la population qu'ils desservent, les CCJ permettent aux jeunes de développer des habiletés de leadership (Jansen, 2001). Ce double bénéfice des comités consultatifs se retrouve souvent dans le discours des organismes qui travaillent auprès des jeunes. Par contre, les bienfaits des CCJ ainsi que les défis qu'ils posent font l'objet de très peu de recherches.

\section{Mon intérêt pour les CCJ}

L'adolescence que j'ai vécue m'a amenée à m'impliquer dans les CCJ et poussée plus tard à entreprendre des études dans le domaine du service social. À 16 ans, j'ai réalisé que je m'identifiais comme allosexuelle ${ }^{1}$. Cette découverte de mon orientation sexuelle a déclenché une remise en question non seulement par rapport à moi-même, mais aussi en lien avec mes réseaux sociaux. En même temps, mon noyau familial était en période de rupture et j'étais perçue comme égoïste en ajoutant du stress pour mes parents qui, à ce moment de ma vie, n'acceptaient pas comme légitime mon orientation sexuelle. Malheureusement, la travailleuse sociale de mon école secondaire n'avait pas à sa disposition en 2003 de ressources en français pour les jeunes LGBTTQ. J'ai donc été dirigée vers une institution bilingue, mais qui n'offrait que des services unilingues anglais à la communauté allosexuelle. Puisque je ne voulais pas me joindre à leurs groupes de soutien, on m'a 
"Ce CCJ auquel j'ai participé adolescente empruntait beaucoup d'idées à l'intervention structurelle. Notamment, nous donner un espace pour revendiquer nos droits comme jeunes allosexuels nous permettait de rendre collectifs nos problèmes individuels.... Le CCJ m'a permis de me créer un espace de réflexion qui m'a poussée à resituer l'homophobie dans un contexte de niveau macro, et m'a permis de distancer mon expérience sur le plan individuel.» recommandé de poser ma candidature pour un poste dans le comité consultatif des jeunes allosexuelles.

À la suite d'une entrevue, j'ai obtenu un poste sur le CCJ qui revendiquait pour des services LGBTTQ pour les jeunes dans la région d'Ottawa. J'aimerais bien dire que ce qui m'a attirée au CCJ était l'idée de revendiquer et que ma vie de jeune militante a débuté ce jour-là, mais ce n'est pas le cas. Seul le sens d'appartenance qu'il m'offrait motivait ma participation au CCJ. Mon expérience passée comme membre d'un CCJ et l'observation de mes collègues et des jeunes avec qui j'y travaille me permettent d'affirmer, et ce, en lien avec la littérature, que le sentiment d'appartenance est la raison principale qui pousse les jeunes à participer à un tel comité. En fait, les recherches indiquent que l'implication de ces derniers influence directement leur sentiment d'appartenance, ce qui a des effets positifs sur leur conception identitaire, leur estime de soi et leur participation dans la communauté (Zeldin, 1995). Le CCJ m'a permis de partager des moments de vie avec des gens qui comprenaient ma réalité, dans une période de vie où j'étais incomprise par mon noyau familial et mon réseau social. En fait, tous les jeunes qui siégeaient au comité avec moi avaient eux aussi vécu des ruptures avec leurs réseaux sociaux et étaient indignés par rapport à la manière dont les jeunes LGBTTQ étaient perçus dans la communauté. J'ai observé la même réalité dans le CCJ à mon travail alors que les participants sont majoritairement des jeunes qui ont vécu des injustices dans les services en santé mentale. Je constate alors que les CCJ offrent des espaces sécuritaires pour qu'ils expriment leur indignation et trouvent des pistes de solution pour effectuer des changements sociaux.

Ce CCJ auquel j'ai participé adolescente empruntait beaucoup d'idées à l'intervention structurelle. Notamment, nous donner un espace pour revendiquer nos droits comme jeunes allosexuels nous permettait de rendre collectifs nos problèmes individuels. Les CCJ utilisent les principes de l'action collective en permettant aux jeunes d'identifier des lacunes dans leurs communautés et de créer des activités, des événements ou des mobilisations sociales afin de faire de l'éducation populaire ou de revendiquer leurs 
droits. Le CCJ m'a permis de me créer un espace de réflexion qui m'a poussée à resituer l'homophobie dans un contexte de niveau macro, et m'a permis de distancer mon expérience sur le plan individuel. Cette participation au CCJ m'a permis de réaliser que la réaction de mon entourage face à ma sexualité était le produit des perceptions sociales véhiculées par l'hégémonie. Ce n'est que récemment que j'ai su nommer cette oppression à l'aide du modèle de Thompson (2006), en comprenant l'interaction entre les plans individuel, culturel et structurel. Mon cheminement dans le CCJ m'a permis d'entreprendre la réflexion et de poser des gestes bien avant ma compréhension académique du problème.

\section{Quelques limites des CCJ}

"Bien que je sois de l'avis que les CCJ ont le potentiel de créer des espaces qui permettent aux jeunes d'être des acteurs sociaux au même titre que les adultes, mes observations et mon vécu m'ont fait réaliser que cette conception des CCJ est utopique et que le pouvoir des jeunes est souvent limité par les adultes de l'institution, qui sont tout aussi influencés par la conception majoritaire des jeunes comme récipiendaires de services. »
Tout d'abord, les CCJ visent à permettre aux jeunes d'acquérir des connaissances et des habiletés qu'ils vont pouvoir utiliser dans leur vie. Entre autres, les CCJ prônent l'entraide, le travail d'équipe, la prise de parole ainsi que l'autonomie (Jansen, 2000). Ces vertus s'alignent avec les caractéristiques recherchées des adultes "fonctionnels" de notre société; ce sont des habiletés qui semblent perçues comme absentes, ou presque, chez les adolescentes et les adolescents. Gordon (2008) avance toutefois que les CCJ s'opposent à la pensée hégémonique voulant qu'ils ne soient importants que parce qu'ils s'orientent vers un futur meilleur. Dans les faits, en créant des espaces où les jeunes peuvent s'exprimer et revendiquer leurs droits, les institutions appuient l'idée que les jeunes sont des acteurs importants et ne sont pas seulement bénéficiaires de services. Bien que je sois de l'avis que les CCJ ont le potentiel de créer des espaces qui permettent aux jeunes d'être des acteurs sociaux au même titre que les adultes, mes observations et mon vécu m'ont fait réaliser que cette conception des CCJ est utopique et que le pouvoir des jeunes est souvent limité par les adultes de l'institution, qui sont tout aussi influencés par la conception majoritaire des jeunes comme récipiendaires de services. 
Pour illustrer cette prémisse, je vais évoquer l'expérience qui m'a fait quitter le CCJ après y avoir œuvré pendant cinq ans. Le comité dans lequel je siégeais avait identifié le besoin d'une retraite à l'extérieur de la ville pour les jeunes LGBTTQ et leurs alliés. Ce camp a permis non seulement aux jeunes de réseauter avec d'autres jeunes allosexuels, mais il offrait aussi des ateliers qui comblaient les lacunes en matière de justice sociale et d'éducation pour la communauté LGBTTQ. En fait, notre comité a mis au programme plusieurs ateliers animés par des jeunes et pour des jeunes. Entre autres, certains sujets abordés traitaient de l'hétérosexisme, de l'éducation sexuelle pro-allosexuelles ainsi que de l'identité de genre qui dépassait la conception binaire homme-femme. Pour animer ces ateliers et permettre la tenue du camp, le CCJ a soumis une demande de financement, a organisé les ateliers et vu à sa planification. Ce séjour a connu un tel succès que nous avons reçu le financement pour tenir le camp une deuxième année. La seule adulte présente était l'employée qui s'occupait de notre comité au sein de l'organisme. Un jour, alors que nous étions en pleine démarche de demande de fonds pour tenir le camp une troisième année, l'employée nous a rassemblés pour nous annoncer que ce dernier sera désormais pris en charge par un autre organisme et qu'il sera animé par des intervenantes et des intervenants adultes. L'employée du CCJ nous a dit que nous étions chanceux et chanceuses puisque nous aurions la priorité pour participer à ce camp. On nous a expliqué que notre projet avait soulevé un besoin dans la communauté, mais qu'il serait plus avantageux s'il était dirigé par des adultes. Par contre, ce "nouveau » camp avait un mandat complètement différent du nôtre qui était une occasion pour des enfants issus de familles allosexuelles ainsi qu'aux jeunes allosexuels de se rassembler.

Après avoir dirigé le camp pendant deux ans, nous étions relégués à la marge et on nous disait, indirectement, que les adultes étaient mieux placés pour comprendre notre vécu de jeunes LGBTTQ $^{2}$. Ce geste était hypocrite venant d'une institution qui se vantait de consulter les jeunes pour sa programmation. Je ne comprenais pas vraiment toutes les implications, mais j'avais compris ceci : cette manière de faire représentait une contradiction 
"J'ai pu voir comment les décisions se prennent en coulisse et comment les suggestions des jeunes sont discréditées, ces derniers étant perçus comme ne sachant pas ce qui est mieux pour eux. " entre la théorie et la pratique des CCJ. Ce malaise par rapport à l'organisme et à son non-respect du processus de consultation - notre comité n'était même pas au courant de la formation de cette nouvelle initiative, même si elle s'adressait aux jeunes allosexuels - m'a fait quitter le CCJ.

Ce n'est que cette année que j'ai repensé à cet événement lors de mon stage avec un CCJ.J'ai pu voir comment les décisions se prennent en coulisse et comment les suggestions des jeunes sont discréditées, ces derniers étant perçus comme ne sachant pas ce qui est mieux pour eux. La conception de leur identité par les adultes crée aussi des impasses par rapport à leur crédibilité comme acteurs sociaux. Par exemple, un groupe de jeunes qui revendique des services en santé mentale peut être étiqueté comme étant incapable de prendre des décisions rationnelles. De plus, des jeunes allosexuels vivant dans une situation économique précaire sont souvent qualifiés de marginaux et discrédités du fait que certains n'adhèrent pas aux valeurs dominantes.

\section{Pistes de réflexion}

"Ma participation dans un CCJ m'a permis de développer une conscience sociale qui a fait de moi une militante pour la justice sociale."
Bien que cette réflexion montre certaines de mes appréhensions par rapport aux CCJ, j'aimerais réitérer le fait que je crois profondément qu'ils ont le potentiel d'être un outil structurel important. Ma participation dans un CCJ m'a permis de développer une conscience sociale qui a fait de moi une militante pour la justice sociale. Je crois que les membres des CCJ peuvent contribuer à des changements positifs et durables dans leurs communautés (Campbell et Erbstein, 2012). Par ailleurs, j'ai vu la grande différence entre un comité dirigé par un jeune versus un CCJ dirigé par une personne adulte. Je crois que les bienfaits et les limites des CCJ peuvent être grandement influencés par la manière dont ceux-ci sont gérés, ce qui peut changer d'un organisme à l'autre. Entre autres, lorsqu'on permet aux jeunes de diriger les réunions du CCJ, on réduit la hiérarchie qui est présente entre un employé rémunéré et les jeunes bénévoles du CCJ. Cela permet aussi que les besoins des jeunes soient pris en compte, 
"Je demeure optimiste à l'idée que le CCJ peut être un outil important dans l'intervention structurelle si celui-ci est utilisé de manière honnête et si les jeunes, leurs idées et leurs besoins sont respectés par l'organisme pour laquelle ils travaillent. Dans cette optique, j'avance que l'idée de rémunérer les jeunes pour leur participation dans les CCJ permet aussi à l'organisme de reconnaître que leurs opinions sont valables et qu'elle a besoin d'eux dans la planification de ses activités et de l'orientation de son travail.» puisque ces derniers ont le pouvoir de décider de l'orientation des réunions et des activités.

Je comprends aussi qu'un comité consultatif demeure un comité de consultation et que cela ne signifie pas que tout ce qui est avancé doit être retenu par l'organisme. Par contre, pour œuvrer dans le paradigme structurel et s'assurer que les jeunes et leurs besoins soient respectés, il serait nécessaire que les organismes soient plus transparents avec les membres de leurs CCJ. Entre autres, dans l'exemple que j'ai cité de mon expérience sur le CCJ, il aurait fallu que le comité soit informé beaucoup plus tôt des intentions de tenir un camp permanent dans la région et que les jeunes soient invités dès le début de cette initiative à y participer. La déception que j'ai vécue provenait du fait que cette nouvelle version du camp nous a été présentée lorsque tout était finalisé et que l'on s'attendait à ce que nous ne soyons plus que de simples "bénéficiaires" de cette initiative. J'aurais certainement été contente de savoir que cette nouvelle initiative permettait d'offrir des services à long terme pour la communauté LGBTTQ dans la région. Lors de mon stage, j'ai vu comment les jeunes peuvent être perçus comme ne méritant pas de partager la vérité, ou étant trop immatures pour comprendre des décisions " adultes ».Je crois que cette conception que les organismes ont de ne pas être obligés de partager les vraies choses avec les membres de leur CCJ est fautive; elle semble aller à l'encontre des principes du CCJ voulant que les jeunes soient les mieux placés pour comprendre les réalités de leurs communautés. Afin de s'assurer d'agir dans une perspective structurelle dont bénéficieraient et l'organisme et les jeunes, il serait nécessaire que le fonctionnement du CCJ soit davantage collaboratif entre les adultes employés et les jeunes qui siègent.

Je demeure optimiste à l'idée que le CCJ peut être un outil important dans l'intervention structurelle si celui-ci est utilisé de manière honnête et si les jeunes, leurs idées et leurs besoins sont respectés par l'organisme pour laquelle ils travaillent. Dans cette optique, j'avance que l'idée de rémunérer les jeunes pour leur participation dans les CCJ permet aussi à l'organisme de reconnaitre que leurs opinions sont valables et qu'elle a besoin d'eux dans la planification de ses activités et de l'orientation de 
son travail.Je comprends aussi que ce n'est pas tous les organismes qui ont de tels moyens, mais je vois cette rémunération comme nécessaire, même si elle se manifeste autrement que par une compensation financière. À titre d'exemple, l'offre d'activités et de formations dont bénéficient les membres du CCJ permettrait la réciprocité entre l'organisme et les jeunes.

Je déclare aussi que c'est la responsabilité des intervenantes et des intervenants qui travaillent directement avec les membres du CCJ de revendiquer pour les intérêts des jeunes. Tout d'abord, ce sont eux qui font le pont entre l'organisme et les jeunes. C'est aussi - malheureusement - l'intervenante ou intervenant qui a le plus de crédibilité auprès des gestionnaires de l'organisme et qui devrait militer pour un changement de vision par rapport au rôle de son CCJ.

\section{Conclusion}

"...les CCJ ont un énorme potentiel de créer des changements structurels si les organismes adhèrent à l'idée que ce sont les jeunes qui sont les experts de leur vie et s'ils agissent de manière à considérer ces derniers comme des acteurs sociaux importants. "
En guise de conclusion, j'estime que l'intervenante ou l'intervenant adulte devrait se fier au fait que les besoins exprimés par les jeunes sont importants. En outre, c'est la responsabilité de l'intervenante ou intervenant de s'assurer que les besoins des jeunes soient respectés par l'organisme. En plus, l'intervenant social devrait permettre aux jeunes d'affirmer leur pouvoir et revendiquer leurs droits tout en s'assurant d'être transparent par rapport aux intentions de l'organisme. Enfin, les CCJ ont un énorme potentiel de créer des changements structurels si les organismes adhèrent à l'idée que ce sont les jeunes qui sont les experts de leur vie et s'ils agissent de manière à considérer ces derniers comme des acteurs sociaux importants. Après tout, le CCJ m'a permis de développer une conscience critique qui m'a permis de voir certaines pistes de changement dans mon lieu de stage. N'est-ce pas là un des postulats de l'intervention structurelle? De permettre le développement d'une conscience critique et de favoriser une démarche collective pour le changement sur le plan politique ou social?... 


\section{Notes}

1 J'utilise le concept allosexuel, qui est un néologisme d'origine québécoise. Ma conception d'allosexuel est interchangeable avec le terme " communauté LGBTTQ " tout au long de cette réflexion.

2 Mon but n'est pas de dire que le nouveau camp à Ottawa est mauvais et que le fait d'avoir des dirigeants adultes est nécessairement néfaste. Je n'ai jamais assisté ou participé à cette initiative et j'ai entendu plusieurs choses positives au sujet de ce camp. J'utilise cet exemple pour illustrer qu'en fait, les jeunes sont souvent discrédités et qu'ils sont perçus comme des bénéficiaires passifs des décisions des adultes. Nous sommes socialisés à accepter les décisions des adultes, peu importe les impacts que cela peut avoir sur les jeunes.

\section{Bibliographie}

CAMPBELL, David, et Nancy ERBSTEIN (2012). «Engaging youth in community change: three key implementation principles ", Community Development, Vol. 43, № 1, p. 63-79.

HOHENEMSER, Lisa, et Beth MARSHALL (2002). «Utilizing a youth development framework to establish and maintain a Youth Advisory Committee ", Health Promotion Practice, p. 155-165.

JANSEN, Karl (2001). «Tapping teens: New Berlin's youth board aims to get kids involved, generate fresh ideas ", Milwaukee Journal Sentinel, p. 51-63.

THOMPSON, Neil (2006). "Chapter 2:The theory base » dans Neil Thompson, Anti-Discriminatory Practice, New York, Palgrave Macmillan, p. 21-46.

THURSTON,Wilfred, et collab. (2004). «Hearing silenced voices : developing community with an advisory committee ", Development in Practice, Vol. 14, No 4, p. 481-494.

ZELDIN, Shepherd (1995). "Community-university collaborations for youth development : From theory to practice ", Journal of Adolescent Research,Vol. 104, N 4, p. 449-469. 